\author{
(อ К.Е.Зоткина ${ }^{1,3}$, О.М.Лесняк ${ }^{2,3}$, А.Ю.Кочиш ${ }^{4}$, И.В.Сушков ${ }^{4}$
}

1 ФГБУ «Национальный медицинский исследовательский центр им. В.А. Алмазова» Минздрава России, Санкт-Петербург, Россия

${ }^{2}$ ФГБОУ ВО «Северо-Западный государственный медицинский университет им. И.И. Мечникова» Минздрава России,

Санкт-Петербург, Россия

${ }^{3}$ ГБУЗ «Клиническая ревматологическая больница № 25», Санкт-Петербург, Россия

${ }^{4}$ ФГБУ «Российский ордена Трудового Красного Знамени научно-исследовательский институт травматологии и ортопедии им. Р.Р. Вредена» Минздрава России, Санкт-Петербург, Россия

Бисфосфонаты являются препаратами первой линии при лечении остеопороза. В последнее десятилетие участилось количество публикуемых случаев о развитии атипичных переломов бедренной кости (АПБК) на фоне длительного лечения бисфосфонатами. В данной статье проведен анализ литературы, посвященной этой проблеме, выделены диагностические критерии АПБК, а также приведен случай АПБК у пациентки, находившейся на терапии алендронатом в течение 3,5 лет.

У пациентки 78 лет, получавшей пероральный бисфосфонат по поводу тяжелого постменопаузного остеопороза, через 3.5 года лечения внезапно появились боли в правом бедре при ходьбе. Через три месяца при падении с высоты собственного роста произошел перелом средней трети правой бедренной кости, по результатам инструментальных исследований имевший все признаки атипичного перелома бедра. Был проведен блокированный интрамедуллярный остеосинтез штифтом. Ретроспективный анализ результатов магнитно-резонансной томографии мягких тканей правого бедра, проведенной до перелома показал наличие недиагностированного неполного перелома средней трети правого бедра, который в последующем реализовался в полный перелом.

Данное клиническое наблюдение демонстрирует сложность диагностики АПБК. Цель публикации - привлечь внимание специалистов к проблеме такого редкого побочного эффекта терапии бисфосфонатами, как АПБК.

КЛЮЧЕВЫЕ СЛОВА: Остеопороз; Алендронат; Переломы бедренной кости; Атипичный перелом; Клинический случай

\title{
A CASE OF ATYPICAL FEMUR FRACTURE DURING LONG-TERM TREATMENT WITH BISPHOSPHONATES IN PATIENT WITH POSTMENOPAUSAL OSTEOPOROSIS
}

\author{
(c) Kira E. Zotkina ${ }^{1,3}$, Olga M. Lesnyak ${ }^{2,3}$, Aleksandr Yu. Kochish ${ }^{4}$, Ivan V. Sushkov ${ }^{4}$
}

\section{${ }^{1}$ Almazov National Medical Research Centre, Saint-Petersburg, Russia}

${ }^{2}$ North-western State Medical University named after I.I. Mechnikov, Saint-Petersburg, Russia

${ }^{3}$ Clinical Rheumatology Hospital N25, Saint-Petersburg, Russia

${ }^{4}$ Vreden Russian Order of Red Banner of Labor Research Institute of Traumatology and Orthopedics, Saint-Petersburg, Russia

Bisphosphonates is a first-line therapy for treatment of osteoporosis. In the last decade, the number of atypical femur fracture (AFF) cases during long-term treatment with bisphosphonates has increased. The aim of this article was to analyze the literature data on this problem, to define the diagnostic criteria of AFF and to present the case of AFF in the patient who received treatment with alendronate for 3.5 years.

A 78-year-old woman, receiving oral bisphosphonate for severe postmenopausal osteoporosis for 3.5 years, suddenly started feeling pain in her right thigh while walking. Three months later, she had got a fracture in middle third of the right femur after falling from her standing height. According to instrumental diagnostics, this fracture had all criteria of AFF. Blocking intramedullary osteosynthesis with shafts was performed. A retrospective analysis of soft tissue magnetic resonance imaging in the area of right thigh, done before the fracture, showed the presence of undiagnosed incomplete right femur fracture in the middle third, which subsequently led to a complete fracture.

Presented clinical case demonstrates the complexity of AFF diagnostics. The purpose of the publication is to draw attention of medical specialists to the issue of this rare side effect of bisphosphonate treatment.

KEYWORDS: Osteoporosis; Alendronate; Femur fractures; Case report 


\section{АКТУАЛЬНОСТЬ}

Остеопороз является системным заболеванием скелета, характеризующимся снижением костной массы и нарушением ее качества (микроархитектоники), что приводит к повышенной хрупкости костей, проявляющейся переломами даже при незначительной травме. Наиболее типичными для остеопороза являются компрессионные переломы тел позвонков, переломы проксимального отдела бедренной кости (шейки и вертельной области), а также проксимального отдела плечевой кости и дистального отдела костей предплечья [1, 2].

Для фармакотерапии остеопороза во всем мире наиболее часто назначаются антирезорбтивные костные препараты из группы бисфосфонатов (БФ), применяющиеся в клинической практике уже около 50 лет. Их эффективность в отношении повышения минеральной плотности костной ткани и предупреждения типичных остеопорозных переломов доказана множеством качественных клинических исследований $[3,4$, 5,6 , 7]. Однако, начиная с 2005 года [8], в специальной литературе появляется все больше публикаций о случаях развития на фоне длительного лечения бисфосфонатами диафизарных переломов бедренной кости, локализация которых не характерна для низкоэнергетических переломов костей, связанных с остеопорозом $[9,10]$.

По современным представлениям к атипичным переломам бедренной кости (АПБК) относят диафизарные переломы на уровне дистальнее малого вертела и проксимальнее дистальных надмыщелков бедренной кости, развившиеся при низкоэнергетической травме или без таковой на фоне длительной (более трех лет) терапии антирезорбтивными костными препаратами, чаще всего - бисфосфонатами. При этом исключаются переломы шейки бедренной кости, большого и малого вертелов и вертельной зоны, переломы, ассоциированные с эндопротезированием крупных суставов, а также патологические переломы, связанные с первичными или метастатическими опухолями костной ткани или различными заболеваниями костей (например, болезнью Педжета или фиброзной дисплазией). Согласно специальному докладу American Society for Bone and Mineral Research (ASBMR), опубликованному в 2014 году, для АПБК, помимо определенной локализации, характерны также специфические «большие» и «малые» клинические критерии, представленные в таблице 1 [11]. При этом для постановки соответствующего диагноза должны присутствовать 4 из 5 «больших» критериев, а «малые» критерии также могут присутствовать у пациента, но не являются обязательными.

Следует отметить, что в отечественной научной литературе встречаются лишь единичные обзорные публикации, посвященные рассматриваемому вопросу $[12,13]$, в которых не содержится описаний собственных клинических случаев. Поэтому характерное клиническое наблюдение, описанное и обсужденное в настоящей статье, может представлять, по мнению авторов, интерес для специалистов различного профиля, занимающихся лечением пациентов с остеопорозом.
Таблица 1. Критерии диагноза «Атипичный перелом бедренной кости»

\section{Большие критерии}

1. Перелом возникает при незначительной травме (падение с высоты собственного роста) или ее отсутствии.

2. Линия перелома берет начало в латеральной части кортикального слоя бедренной кости и имеет поперечную ориентацию, но, удлиняясь, может идти также и косо.

3. Полный перелом проходит через латеральный и медиальный кортикальные слои бедренной кости и может образовать медиальную «шпору», или «спикулу»; а неполный перелом затрагивает только латеральный кортикальный слой.

4. Перелом не имеет мелких костных отломков или включает минимальное их количество.

5. Имеется конусообразное или клювовидное утолщение надкостницы и эндоста латерального кортикального слоя бедренной кости в месте перелома.

\section{Малые критерии}

1. Увеличение толщины кортикального слоя бедренной кости в районе диафиза.

2. Продромальный синдром в виде болей ноющего характера в области будущего перелома.

3. Замедленный период консолидации.

4. Двусторонний неполный или полный перелом диафиза бедренной кости.

\section{ОПИСАНИЕ СЛУЧАЯ}

У пациентки К., 1940 года рождения, проживающей в Санкт-Петербурге, в 2008 году были выявлены спонтанные компрессионные переломы тел двух позвонков (Th12 и L1) неизвестной давности. В 2012 году у нее произошел типичный для остеопороза низкоэнергетический перелом дистального метаэпифиза лучевой кости, однако специфической антиостеопоротической терапии она не получала.

В апреле 2014 года на основании данных анамнеза и результатов двухэнергетической рентгеновской абсорбциометрии (ДРА) у пациентки был диагностирован тяжелый постменопаузный остеопороз, осложненный множественными компрессионными переломами тел позвонков и низкоэнергетическим невертебральным переломом. Пациентка начала прием алендроната в комбинации с холекальциферолом (70 мг/140 мкг в неделю) и препаратами кальция (1000 мг в сутки) и продолжала лечение на протяжении 3,5 лет до сентября 2017 года. Результаты ДРА на аппарате Lunar iDXA в период приема алендроната приведены в таблице 2 .

Сравнение данных ДРА в динамике показывает, что в первый год приема антиостеопоротической терапии МПКТ и Т-критерий в поясничных позвонках у пациентки К. практически не изменились, но через 3 года и 5 месяцев приема алендроната была отмечена отчетливая положительная динамика в значениях указанных показателей. При этом в сентябре 2017 года по данным лабораторных тестов уровни ионизированного кальция 
Таблица 2. Показатели минеральной плотности костной ткани (МПКТ) и Т-критерия по результатам ДРА у пациентки К. в динамике в период приема алендроната

\begin{tabular}{lcccccc}
\hline Дата & \multicolumn{2}{c}{$\mathbf{0 1 . 0 4 . 2 0 1 4}$} & \multicolumn{2}{c}{$\mathbf{2 5 . 0 6 . 2 0 1 5}$} & \multicolumn{2}{c}{$\mathbf{2 2 . 0 8 . 2 0 1 7}$} \\
\hline L1 & МПКТ (г/см2) & Т-критерий & МПКТ (г/см2) & Т-критерий & МПкт (г/см2) & Т-критерий \\
L2 & 0,852 & $-2,4$ & 0,815 & $-2,7$ & 0,904 & $-1,9$ \\
L3 & 0,646 & $-4,7$ & 0,689 & $-4,3$ & 0,725 & $-4,0$ \\
L4 & 0,812 & $-3,2$ & 0,729 & $-3,4$ & 0,885 & $-2,7$ \\
Total & 0,839 & $-3,0$ & 0,853 & $-2,8$ & 0,912 & $-2,4$ \\
\hline
\end{tabular}

и фосфора в крови пациентки определялись в пределах референсных значений, а уровень щелочной фосфатазы (ЩФ) (55 ед/л) также был в пределах нормы (от 30 до 117 ед/л). Поэтому ей было рекомендовано продолжать назначенную антиостеопоротическую терапию.

Однако 30 августа 2017 года пациентка споткнулась на улице и на вершине напряжения мышц почувствовала резкую боль в верхней половине правого бедра. Несмотря на выраженный болевой синдром, она самостоятельно дошла до дома. К врачу не обращалась, использовала для местного лечения различные мази с нестероидными противовоспалительными препаратами (НПВП). При этом боль тупого ноющего характера постоянно возникала при ходьбе локально в верхней половине левого бедра, а в состоянии покоя купировалась.

Через две недели в связи с сохранением болевого синдрома пациентка обратилась к хирургу в поликлинику по месту жительства, где клинически был диагностирован артроз правого тазобедренного сустава, но дополнительные исследования не проводились. Была назначена терапия пероральными НПВП и магнитотерапия (местно), но характерный болевой синдром в правом бедре сохранялся.
В ноябре 2017 года с теми же жалобами пациентка обратилась к травматологу-ортопеду в поликлинику по месту жительства. Ей была выполнена рентгенография правого тазобедренного сустава, по данным которой были выявлены признаки коксартроза 2-ой стадии. Тогда же пациентке была проведена магниторезонансная томография (МРТ) правого бедра. По результатам этого исследования было описано лишь умеренное повышение МР-сигнала в средней трети диафиза правой бедренной кости и минимальный отек мягких тканей в этой области. Однако при ретроспективной оценке (в октябре 2018 года) результатов указанного МРТ-исследования специалистом в области лучевой диагностики из РНИИТО имени Р.Р. Вредена была выявлена характерная картина незавершенного диафизарного перелома правой бедренной кости, встречающаяся при атипичных переломах на фоне длительного приема бисфосфонатов. Приводим далее соответствующие срезы МРТ (Рис. 1 а, б, в) и заключение специалиста.

В средней трети диафиза правой бедренной кости по латеральной стороне отмечается локальное утолщение кортикального слоя: максимально - до 10,2 мм, шириной до 11,7 мм и на протяжении 19,3 мм за счет частично

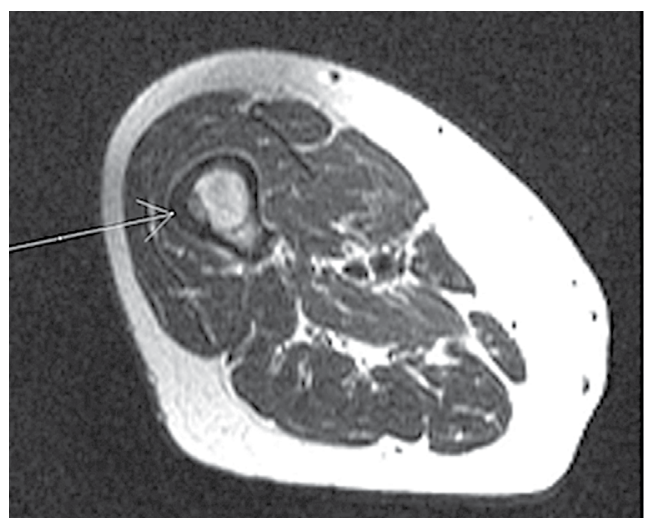

A

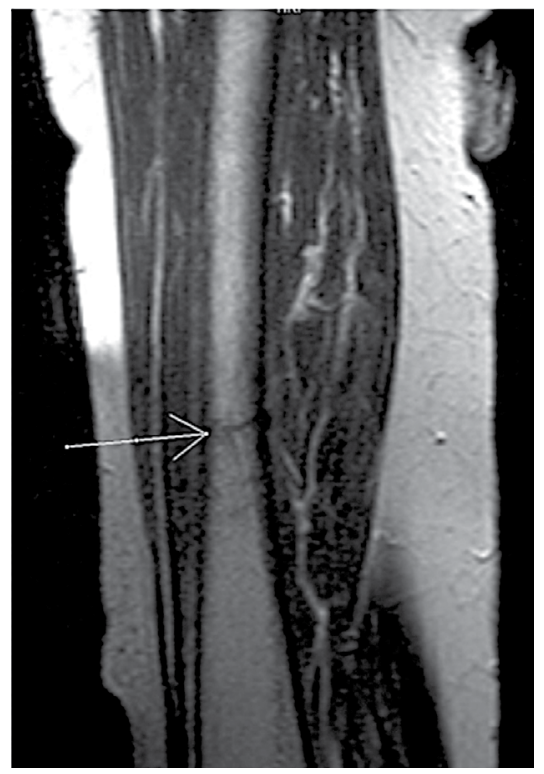

Б

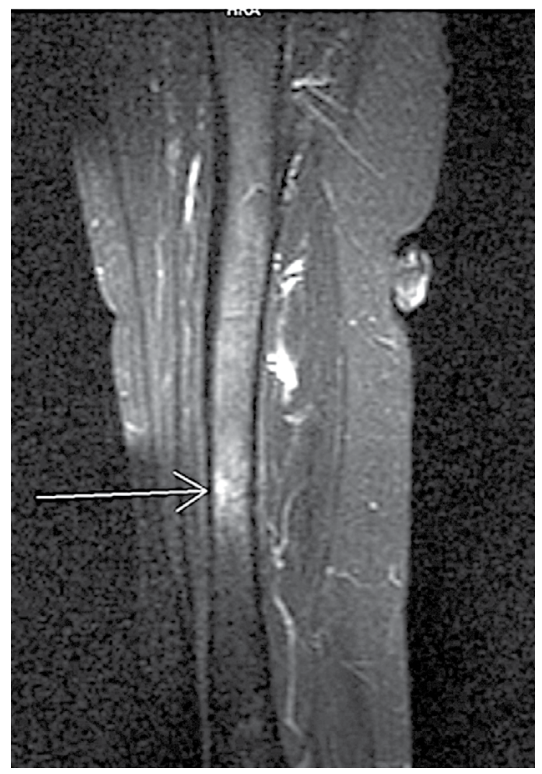

B

Рис. 1. Показательные срезы МРТ-исследования правого бедра пациентки К. от 09.11.2017 г. Примечание:

а) локальное утолщение кортикального слоя правой бедренной кости за счет частично ассимилированных эндостальных наслоений; б) прерывание контура кортикального слоя бедренной кости в центе описываех изменений с наличием неполного по поперечнику щелевидного дефекта костной структуры толщиной до 2 мм, протяженностью по ширине поперечника кости до 16,3 мм;

в) зона реактивного отека костного мозга бедренной кости шириной до 45,1 мм в месте описываемых изменений. 
ассимилированных эндостальных наслоений (Рис. 1а). Определяется прерывание контура кортикального слоя кости в центе описанных изменений с наличием неполного по поперечнику щелевидного дефекта костной структуры толщиной до 2 мм, протяженностью по ширине поперечника кости до 16,3 мм, проявляющегося гипоинтенсивным сигналом на T2, T1 и PD-FS (Рис. 16), с зоной реактивного отека костного мозга бедренной кости шириной до 45,1 мм (Рис. 1в). Целостность кортикального слоя бедренной кости на остальном протяжении сохранена. Мягкие ткани бедра без признаков патологических изменений. В визуализируемых зонах контралатерального (левого) бедра признаков патологических изменений не выявлено.

К сожалению, эти весьма характерные изменения не были замечены и описаны в ноябре 2017 года. Поэтому пациентке К. был подтвержден диагноз остеоартроза правого тазобедренного сустава 2 стадии и назначена терапия пероральными НПВП и витаминами группы В, которая не дала положительного эффекта.

Через 3 месяца после возникновения болей в области правого бедра - в декабре 2017 года пациентка во время передвижения по своей квартире внезапно почувствовала резкую боль в области правого бедра, нога, с ее слов, «подломилась», она упала и не смогла подняться. Далее она была госпитализирована в городской травматологический стационар, где на основании рентгенографии (Рис. 2) был диагностирован диафизарный перелом правой бедренной кости в средней трети типа 42A1 по классификации Ассоциации остеосинтеза. Пациентке был выполнен интрамедуллярный остеосинтез штиф-

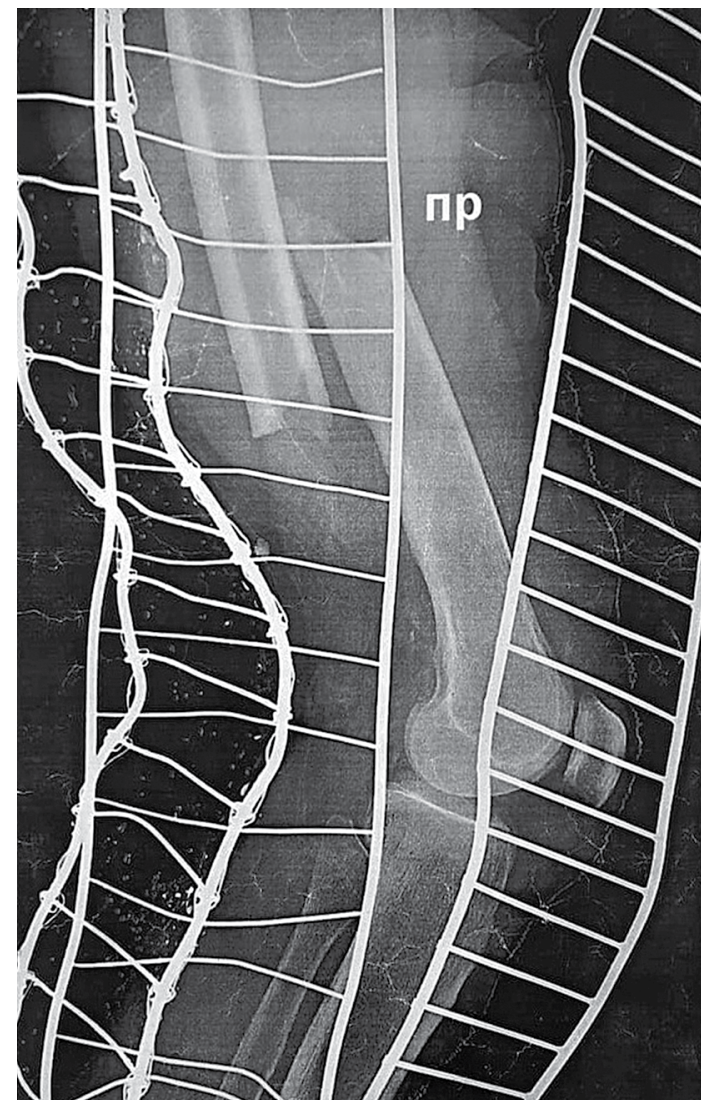

Рис. 2. Рентгенограмма правой нижней конечности пациентки К. в день поперечного диафизарного атипичного перелома правой бедренной кости типа 42A1 по классификации Ассоциации остеосинтеза, от 04.12.2017 года. том с блокированием. При этом прием алендроната был прекращен. Послеоперационный период протекал без осложнений и уже через 8 недель по данным контрольной рентгенографии перелом консолидировался (Рис. 3). Далее через 4 месяца после перелома функция правой нижней конечности была восстановлена практически в полном объеме, а болевой синдром был полностью купирован.

Во время обследования пациентки авторами статьи в октябре 2018 года на основании анализа клинических данных и результатов инструментальных исследований был ретроспективно заподозрен атипичный перелом правой бедренной кости (АПБК) на фоне продолжительного (3.5 года) систематического приема алендроната. По данным лабораторных исследований у нее была выявлена недостаточность витамина D - 23,31 нг/мл (норма 30-150 нг/мл). Однако остальные изученные показатели крови были в пределах референсных значений: общий кальций - 2,05 ммоль/л (референсный диапазон 2,02-2,6 ммоль/л), фосфор - 1,07 ммоль/л (референсный диапазон 0,87-1,45 ммоль/л), щелочная фосфатаза - 56 ед/л (референсный диапазон 30-117 ед/л). Для исключения АПБК вследствие взрослой формы гипофосфатазии пациентке был проведен контроль уровня пиридоксина сыворотки крови, который оказался в норме - 12,58 нг/ мл (референсный диапазон 8,7-27,2 нг/мл).

По результатам обследования пациентке К. была рекомендована костноанаболическая терапия терипаратидом 20 мкг в день подкожно в течение года с последующим контролем денситомтерии в комбинации с препаратами кальция и витамина D (1000 мг/2000 МЕ в сутки).

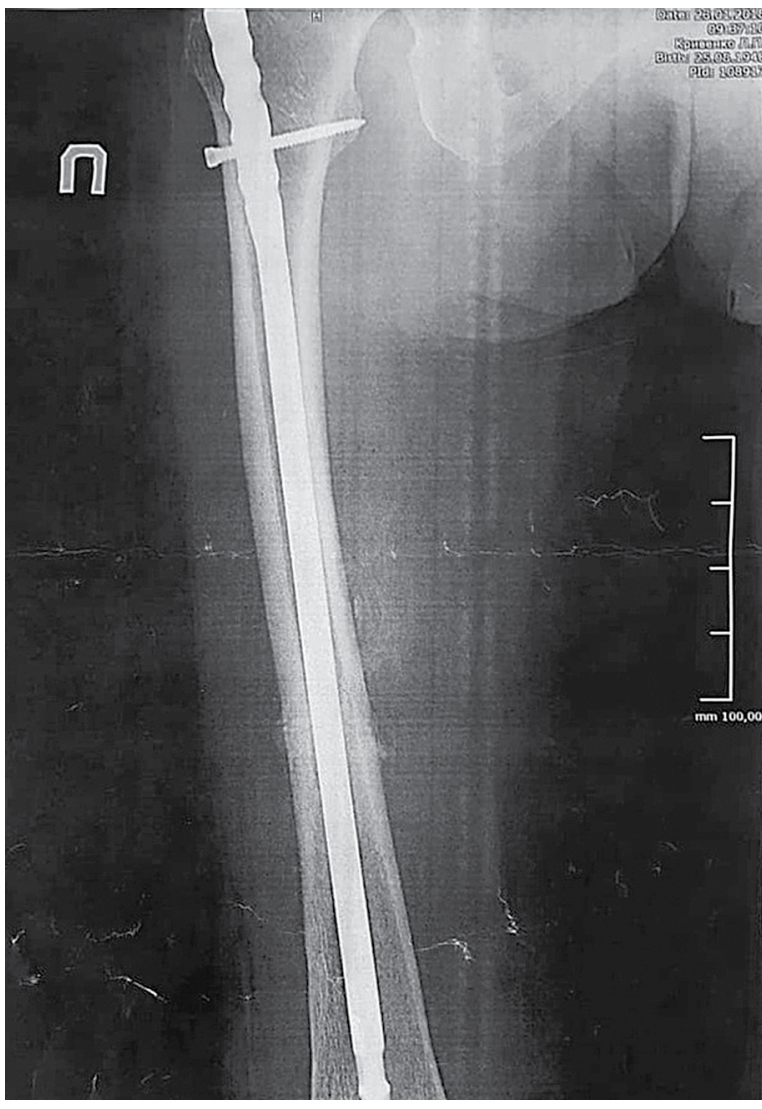

Рис. 3. Рентгенограмма правого бедра пациентки К. через 8 недель после интрамедуллярного остеосинтеза бедренной кости штифтом с блокированием, от 26.01.2018 года. 


\section{ОБСУЖДЕНИЕ}

Со времени первого описания Odvina CV et al., 2005 [8] атипичного перелома бедренной кости (АПБК) на фоне продолжительной терапии бисфосфонатами (БФ) прошло уже почти 15 лет, а количество научных публикаций на эту тему продолжает прогрессивно возрастать. Так, к концу 2018 года в системе PubMed было зарегистрировано уже 603 публикации по ключевым словам «атипичный перелом» и «бисфосфонаты», что говорит о несомненной актуальности обсуждаемой проблемы.

В настоящее время связь АПБК с продолжительным приемом БФ не вызывает сомнений. В отношении патогенеза этой патологии с самого начала было выдвинуто предположение о ее развитии вследствие избыточного подавления костной резорбции на фоне лечения БФ. Как известно, бисфосфонаты ингибирует активность и индуцируют апоптоз остеокластов, что тормозит резорбцию костной ткани и в целом «замораживает» костный обмен. Такое действие, с одной стороны, приводит к росту минеральной плотности костной ткани (МПКТ), но, с другой стороны, ее механическая прочность при этом может снижаться за счет сохранения скомпрометированных костных балок с наличием множества микротрещин $[1,3,4]$. Эта гипотеза подтверждается наблюдениями аналогичных атипичных переломов при гипофосфатазии у взрослых - редком генетическом заболевании, характеризующемся замедленным костным обменом [14], а также при длительном приеме другого (отличного от бисфосфонатов по механизму действия) антирезорбтивного костного препарата - деносумаба [15].

По современным данным риск развития АПБК у людей, принимающих БФ, выше в 1,7-2,7 раза, чем в общей популяции $[6,7]$. В частности, такой риск составлял 1,78/100000 в год для тех, кто получает БФ менее двух лет. В той же популяции риск возрос до 38,9/100000 случаев в год на фоне приема БФ в течение 6-8 лет, и до $107,5 / 100000$ в год у пациентов, принимавших БФ более 10 лет. Однако, следует особо отметить, что большинство авторов пишут о существенном превышении пользы от приема БФ у пациентов с остеопорозом по отношению к риску всех известных побочных эффектов, в том числе АПБК $[3,6,7,11,16]$.

Необходимо также отметить, что для обсуждаемой патологии разработаны достаточно четкие диагностические критерии, представленные во введении нашей статьи, а также созданы клинические рекомендации по предупреждению АПБК и по ведению пациентов после случившихся атипичных переломов $[4,10,11]$. В частности, группа специалистов ASBMR рекомендует следующую стратегию лечения пациентов с АПБК: прервать прием бисфосфонатов (или деносумаба), обеспечить адекватный уровень потребления кальция и витамина $\mathrm{D}$, а также использовать анаболическую костную терапию терипаратидом [11].
Несмотря на большое количество зарубежных научных публикаций, посвященных проблеме атипичных переломов бедренной кости на фоне длительной антирезорбтивной костной терапии, в отечественной литературе эта достаточно редкая патология описана явно недостаточно. В частности, представленное в настоящей статье описание характерного клинического случая у пациентки К., 78 лет, вероятно, является первым в нашей стране выявленным и подробно разобранным клиническим наблюдением. Поэтому многие отечественные врачи не имеют соответствующей научной и клинической информации, что приводит к несвоевременной диагностике и определенным ошибкам в тактике лечения пациентов обсуждаемого профиля. Так, в представленном клиническом наблюдении достаточно характерные и яркие симптомы развивающегося АПБК, а также вполне однозначная картина МРТ-исследования, соответствовавшие всем пяти «большим» диагностическим критериям ASBMR (таблица 1), не были правильно интерпретированы и учтены, что привело к возникновению у пожилой пациентки диафизарного перелома бедренной кости. К счастью, качественно выполненный интрамедуллярный остеосинтез и отмена приема алендроната у пациентки К. позволили срастить перелом в обычные средние сроки и добиться практически полного восстановления функции поврежденной нижней конечности. Назначение ей анаболической костной терапии терипаратидом создает, на наш взгляд, необходимые условия для эффективного лечения тяжелого постменопаузного остеопороза и предупреждения повторных остеопорозных переломов.

\section{ЗАКЛЮЧЕНИЕ}

Представленный и обсужденный клинический случай атипичного перелома бедренной кости у пациентки К., 78 лет, произошедший на фоне длительного (3,5 года) приема алендроната по поводу тяжелого постменопаузного остеопороза, является, на взгляд автором, достаточно ярким и поучительным примером, знакомство с которым может принести практическую пользу врачам различных специальностей в отношении диагностики, профилактики и лечения достаточно редкого, но тяжелого осложнения длительной терапии антирезорбтивными костными препаратами.

\section{ДОПОЛНИТЕЛЬНАЯ ИНФОРМАЦИЯ}

Согласие пациента. Пациентка добровольно подписала информированное согласие на публикацию персональной медицинской информации в журнале «Остеопороз и остеопатии».

Конфликт интересов. Авторы декларируют отсутствие явных и потенциальных конфликтов интересов, связанных с публикацией настоящей статьи 


\section{СПИСОК ЛИТЕРАТУРЫ | REFERENCES}

1. Профилактика, диагностика и лечение дефицита витамина D и кальция среди взрослого населения и у пациентов с остеопорозом. Рекомендации Российской ассоциации по остеопорозу / Под ред. О.М. Лесняк. - М.: ГЭОТАР-Медиа, 2016. [Lesnyak OM, editor. Profilaktika, diagnostika i lecheniye defitsita vitamina D i kal'tsiya sredi vzroslogo naseleniya i u patsiyentov s osteoporozom. Rekomendatsii Rossiyskoy assotsiatsii po osteoporozu. Moscow: GEOTAR-Media; 2016. (In Russ).]

2. Kanis JA, Burlet N, Cooper C, et al. European guidance for the diagnosis and management of osteoporosis in postmenopausal women. Osteoporos. Int. 2008;19(4):399-428. doi: https://doi.org/10.1007/s00198-008-0560-z

3. Schilcher J, Koeppen V, Aspenberg P, Michaëlsson K. Risk of atypical femoral fracture during and after bisphosphonate use. Acta Orthop. 2015;86(1):100-107. doi: https://doi.org/10.3109/17453674.2015.1004149

4. Toro G, Ojeda-Thies C, Calabrò G, et al. Management of atypical femoral fracture: a scoping review and comprehensive algorithm. BMC Musculoskel. Disord. 2016;17(1). doi: https://doi.org/10.1186/s12891-016-1086-8

5. Dell RM, Adams AL, Greene DF, et al. Incidence of atypical nontraumatic diaphyseal fractures of the femur. J. Bone Miner. Res. 2012;27(12):2544-2550. doi: https://doi.org/10.1002/jbmr.1719

6. Gedmintas L, Solomon DH, Kim SC. Bisphosphonates and risk of subtrochanteric, femoral shaft, and atypical femur fracture: A systematic review and meta-analysis. J. Bone Miner. Res. 2013;28(8):1729-1737. doi: https://doi.org/10.1002/jbmr.1893

7. Lee $\mathrm{S}$, Yin RV, Hirpara H, et al. Increased risk for atypical fractures associated with bisphosphonate use. Fam. Pract. 2015;32(3):276-281. doi: https://doi.org/10.1093/fampra/cmu088

8. Odvina CV, Zerwekh JE, Rao DS, et al. Severely Suppressed Bone Turnover: A Potential Complication of Alendronate Therapy. J. Clin. Endocr. Metab. 2005;90(3):1294-1301. doi: https://doi.org/10.1210/jc.2004-0952

9. Lloyd AA, Gludovatz B, Riedel C, et al. Atypical fracture with long-term bisphosphonate therapy is associated with altered cortical composition and reduced fracture resistance. Proceedings of the National Academy of Sciences. 2017;114(33):8722-8727. doi: https://doi.org/10.1073/pnas.1704460114

10. Rizzoli R, Åkesson K, Bouxsein M, et al. Subtrochanteric fractures after long-term treatment with bisphosphonates: a European Society on Clinical and Economic Aspects of Osteoporosis and Osteoarthritis, and International Osteoporosis Foundation Working Group Report. Osteoporos. Int. 2010;22(2):373-390. doi: https://doi.org/10.1007/s00198-010-1453-5

11. Shane E, Burr D, Abrahamsen B, et al. Atypical Subtrochanteric and Diaphyseal Femoral Fractures: Second Report of a Task Force of the American Society for Bone and Mineral Research. J. Bone Miner. Res. 2014;29(1):1-23. doi: https://doi.org/10.1002/jbmr.1998

12. Голубев Г.Ш. Атипичные переломы бедра при длительном лечении бисфосфонатами (критический обзор литературы) // Травматология и ортопедия России. - 2013. - Т.19. - №1. - C.113-123. [Golubev GS. Atypical fractures due to long treatment with bisphosphonates (critical review). Traumatology and Orthopedics of Russia. 2013;19(1):113-123. (In Russ).] doi: https://doi.org/10.21823/2311-2905-2013--1-113-123

13. Кочиш А.Ю., Головач И.Ю. К вопросу о риске атипичных переломов бедренной кости при длительном приеме бисфосфонатов // Травматология и ортопедия России. - 2013. - Т.19. - №1.C. 123 - 126. [Kochish AYu, Golovach IYu. K voprosu o riske atipichnykh perelomov bedrennoy kosti pri dlitel'nom priyeme bisfosfonatov. Traumatology and Orthopedics of Russia. 2013;19(1):123126. (In Russ).]

14. Whyte MP. Atypical Femoral Fractures, Bisphosphonates, and Adult Hypophosphatasia. J. Bone Miner. Res. 2009;24(6):1132-1134. doi: https://doi.org/10.1359/jbmr.081253

15. Aspenberg P. Denosumab and atypical femoral fractures. Acta Orthop. 2013;85(1):1-1. doi: https://doi.org/10.3109/17453674.2013.859423

16. Dell RM, Adams AL, Greene DF, et al. Incidence of atypical nontraumatic diaphyseal fractures of the femur. J. Bone Miner. Res. 2012;27(12):2544-2550. doi: https://doi.org/10.1002/jbmr.1719

\section{ИНФОРМАЦИЯ ОБ АВТОРАХ [AUTHORS INFO]}

*Зоткина Кира Евгеньевна, [Kira E. Zotkina]; e-mail: kira.zotkina@gmail.com; адрес: Россия, Санкт-Петербург, ул. Большая Подъяческая 30 [address:30 Bolshaya Pod'yacheskaya str., 1190068 St.Petersburg, Russia]; ORCID: https://orcid.org/0000-0002-8188-1289; eLibrary SPIN: 3494-9650;

Лесняк Ольга Михайловна, д.м.н., проф. [Olga M. Lesnyak, MD, PhD, Professor]; e-mail: olga.m.lesnyak@yandex.ru; ORCID: https://orcid.org/0000-0002-0143-0614; eLibrary SPIN: 6432-4188;

Кочиш Александр Юрьевич, д.М.H, проф. [Aleksandr Yu. Kochish, MD, PhD, Professor]; e-mail: auk1959@mail.ru; ORCID: https://orcid.org/0000-0002-2466-7120; eLibrary SPIN: 7522-8250;

Сушков Иван Владимирович [Ivan V. Sushkov]; eLibrary SPIN: 9260-1613;

ORCID: https://orcid.org/0000-0002-8439-8321;

\section{ИНФОРМАЦИЯ}

Рукопись получена: 12.07.2019. Одобрена к публикации: 05.08.2019.

\section{КАК ЦИТИРОВАТЬ:}

Зоткина К.Е., Лесняк О.М., Кочиш А.Ю., Сушков И.В. Атипичный перелом бедренной кости на фоне лечения бисфосфонатами пациентки с постменопаузным остеопорозом. // Остеопороз и остеопатии. — 2019. — Т. 22. — №1. — С.18-23. doi: https://doi.org/10.14341/osteo10286

\section{TO CITE THIS ARTICLE:}

Zotkina KE, Lesnyak OM, Kochish AYu, Sushkov IV. A case of atypical femur fracture during long-term treatment with bisphosphonates in patient with postmenopausal osteoporosis. Osteoporosis and bone diseases. 2019;22(1):18-23. doi: https://doi.org/10.14341/osteo10286 